\title{
Heterosexual University Students' Attitudes Toward Same-Sex Couples and Parents Across Seven European Countries
}

\author{
Salvatore D'Amore ${ }^{1}$ (D) $\cdot$ Robin Wollast ${ }^{1} \cdot$ Robert-Jay Green $^{2} \cdot$ Pierre Bouchat $^{3} \cdot$ Pedro Alexandre Costa $^{4}$. \\ Katie Katuzny ${ }^{5}$. Thérèse Scali ${ }^{6} \cdot$ Roberto Baiocco $^{7}$ • Olivier Vecho ${ }^{8} \cdot$ Magdalena Ewa Mijas $^{9} \cdot$ Marta Evelia Aparicio $^{10}$. \\ Klio Geroulanou ${ }^{11} \cdot$ Olivier Klein $^{1}$
}

Accepted: 1 November 2020

(C) Springer Science+Business Media, LLC, part of Springer Nature 2020

\begin{abstract}
Introduction This research examined the general attitudes toward lesbian women and gay men (LG people), same-sex marriage (SSM), and LG parenting (LGP) in a large sample of young heterosexual European adults. We expected that one's country of origin, gender role traditionalism, contact, and religiosity would predict their responses.

Methods We conducted a large-scale study from April 2012 to November 2014. The sample consisted of 13,403 self-identified heterosexual students from Belgium, Italy, France, Portugal, Poland, Spain, and Greece (38.7\% men and 61.2\% women). The main research variables were general attitudes toward LG people, support for same-sex coupling and parenting, gender role traditionalism beliefs, frequency and quality of the contact with LG people, and religiosity.

Results We found that the attitudes toward these issues were significantly more negative in Poland and less negative in Greece than in the other countries included in the sample. In addition, these national differences were explained by psychological variables, mainly religiosity and general attitudes toward LG people. Although participants' higher satisfaction levels regarding their contact with LG people and lower levels of gender role traditionalism were associated with positive attitudes toward SSM and parenting, these factors did not account for the attitudinal variability between these countries.

Conclusions Attitudes toward LG people are changing throughout Europe and are influenced by each country's sociopolitical context. We highlight the societal importance of attitudes toward SSM and LG parenting and recommend facilitating positive social contact in universities and other settings to improve young adult heterosexuals' attitudes.

Policy Implications This study's results can inform policies aiming to reduce inequality and develop more inclusive policies on same-sex parented families and couples.
\end{abstract}

Keywords Attitudes $\cdot$ Lesbian and gay couples $\cdot$ Lesbian and gay parents $\cdot$ Homophobia $\cdot$ Gender role traditionalism $\cdot$ Religiosity . Contact with lesbian and gay people

Salvatore D'Amore sdamore@ulb.ac.be

1 Faculty of Psychological Sciences and Education, Université Libre de Bruxelles, Avenue F.D. Roosevelt 50 - CP 122, 1050 Brussels, Belgium

2 California School of Professional Psychology, Alliant International University, 1 Beach Street, Suite 100, San

Francisco, CA 94133-1221, USA

3 Équipe PErSEUS (EA 7312), UFR Sciences Humaines et Sociales, Université de Lorraine, Nancy, France

4 Instituto Universitario Ciencias Psicologicas, Sociais e da Vida-ISPA, Rua Jardim do Tabaco n³4, 1149-041 Lisbon, Portugal

5 Sexual and Gender Minority Health Equity Lab, University of California, San Francisco, USA
6 Faculty of Psychology, University of Liège, Quartier Agora, Place des Orateurs, 2 (Bât B32), 4000 Liège, Belgium

7 Faculty of Medicine and Psychology, University La Sapienza, Piano IV, stanza 408, Via dei Marsi 78, 00185 Rome, Italy

8 Faculty of Psychology, Université Paris Nanterre, UFR SPSE, Bureau C402200 , Av. de la République, 200, 92001 Nanterre Cedex, France

9 Department of Environmental Health, Jagiellonian University Medical College, Grzegorzecka 20 31-531, Krakow, Poland

10 Faculty of Psychology, Universidad Complutense de Madrid, Campus de Somosaguas, s/n, 28223 Madrid, Spain

11 New York College Athens, Leoforos Vasilis Amalias, 38, 10558 Athens, Greece 
Same-sex couples and parents are becoming more visible across Europe, with a number of equal civil rights being granted to them in the areas of marriage or legalized civil unions/partnerships, access to adoption and medical reproductive services, and parenting/coparenting legal protections. These rights should produce additional positive changes regarding the perceptions of same-sex families in Europe (European Commission, 2015).

As of 2020, 16 European countries (Austria, Belgium, Denmark, Finland, France, Germany, Iceland, Luxembourg, Malta, Netherlands, Portugal, Spain, Sweden, and the UK) have legally recognized and allowed same-sex marriage; and 13 (Andorra, Croatia, Cyprus, Czech Republic, Estonia, Greece, Hungary, Italy, Liechtenstein, Monaco, San Marino, Slovenia, and Switzerland) have recognized civil unions/civil partnerships. Full joint adoption by same-sex couples is legal in 17 European countries (Andorra, Austria, Belgium, Denmark, Finland, France, Germany, Iceland, Ireland, Luxembourg, Malta, Netherlands, Norway, Portugal, Spain, Sweden, and the UK). However, research on the association between legal recognition and attitudes toward same-sex marriage (SSM) or lesbian/gay parenting (LGP) is lacking. Hooghe and Meeusen (2013) found that the levels of sexual prejudice are significantly lower in countries where marriage equality is granted. It is unclear whether the lower rates of prejudice in some countries are the result of, or caused by, the advent of marriage equality.

Studies have revealed diversity in attitudes toward SSM and LGP across Europe (Commissioner for Human Rights, 2011). In relation to the countries included in this research, Belgium, France, and Spain showed the highest levels of acceptance of same-sex couples and parenting, whereas Greece and Poland showed the lowest levels (European Commission, 2015). Attitudes toward SSM and LGP remain unfavorable in most countries, particularly in Eastern Europe (Caroll \& Mendos, 2017); there is still some reluctance to grant custody of a child to same-sex couples, particularly male couples (Averett \& Hedge, 2012).

The most common argument against LGP is based on judgments about immorality or the potentially negative effects of the parents' homosexuality on their children's development (Clarke, Kitzinger, \& Potter, 2004). Neither of the arguments in opposition to SSP have empirical support. These arguments are detrimental not only to same-sex couples and parents but also to gay and lesbian youths and adults not raising children. Research shows that children of same-sex parents are well adjusted and do not suffer from their parents' sexual orientations. Rather, discrimination experienced by LG parents and children may negatively affect the quality of family processes (see Baiocco, Carone, Ioverno, \& Lingiardi, 2018; Carone, Baiocco, Ioverno, Chirumbolo, \& Lingiardi, 2017; Carone, Lingiardi, Chirumbolo, \& Baiocco, 2018; Farr, 2017; Fedewa, Black, \& Ahn, 2015; Gartrell, Bos, \& Koh, 2018; Green, Rubio, Rothblum, Bergman, \& Katuzny, 2019; Rubio et al., 2017).
In our cross-national study, we investigated the attitudes toward SSM and LGP in Belgium, France, Portugal, Italy, Spain, Greece, and Poland. These countries were selected because of their diverse sociopolitical contexts related to LGBT couples and family rights. LGBT rights in these countries are illustrative of the acceptance of SSM and LGP. Building on and extending the findings in the current literature on attitudes toward SSM and LGP, we tested a theoretical model across these seven countries where we compared the levels of support for SSM and LGP. To understand cultural differences, we included sociodemographic factors, such as age and gender, as well as psychological factors, such as religiosity, gender role traditionalism, contact with LG people, and attitudes toward LG people, to explain cultural differences. Table 1 displays the major SSM and LGP rights available in each of the seven countries.

Sexual prejudice is an antecedent of negative attitudes toward SSM and LGP by fostering negative emotional reactions toward sexual minorities (Costa, Carneiro, Esposito, D'Amore, \& Green, 2017; Herek, 2002). Consequently, the prevalence of negative attitudes creates contextual stressors that adversely affect gay people's health and families (Prendergast \& MacPhee, 2018). Research has suggested that religiosity, the traditional gender ideology, and the quantity/satisfaction of contact with LG people all contribute to fostering negative attitudes and discrimination toward LG people as well as toward SSM and LGP (Webb \& Chonody, 2014). One of the most widespread aspects of discrimination toward LG people is the lack of equality in SSM and LGP (Hull, 2003). In particular, public policies that do not legally recognize SSM and LGP sometimes have negative impacts on couple's and family functions, parental legitimization by society in general, and social support from their family of origin, workplaces, and other healthcare, religious, and social settings (Costa et al., 2017).

Although many studies have investigated the sociodemographic and social psychological predictors of attitudes toward homosexuality and same-sex relations, the literature on heterosexuals' attitudes toward SSM and LGP in Europe remains scarce (Costa et al., 2017; Costa \& Davies, 2012; Ioverno et al., 2018). In this study, we examined heterosexual youths' attitudes toward SSM and LGP and their predictors in seven European countries. We considered sociodemographic and social psychological factors, such as gender role traditionalism, religiosity, contact satisfaction with sexual minorities, and attitudes toward LG people.

\section{Attitudes Toward LG People, SSM, and LGP}

A range of variables influence attitudes toward LG people, such as age, gender, level of education, ethnicity, religiosity, conservative political affiliation, beliefs about whether samesex orientation is a choice, and intergroup contact (e.g., Costa 
Table 1 Introduction of same-sex marriage, registered partnership, and joint adoption in seven European countries

\begin{tabular}{llllllll}
\hline & Belgium & France & Greece & Italy & Poland & Portugal & Spain \\
\hline Same-sex marriage & 2003 & 2013 & - & - & - & 2013 & 2005 \\
Registered partnership & 2000 & 1999 (PACS) & 2015 & 2016 & - & 2010 & - \\
Adoption & 2006 & 2013 & - & - & - & 2015 & 2005 \\
\hline
\end{tabular}

et al., 2017). These variables also predict attitudes toward SSM (Brumbaugh, Sanchez, Nock, \& Wright, 2008; Sherkat, De Vries, \& Creek, 2010) and LGP (Costa, Pereira, \& Leal, 2015; Vecho, Gross, Gratton, D’Amore, \& Green, 2018).

Researchers have suggested that age and gender are the strongest sociodemographic predictors of attitudes toward SSM (Brumbaugh et al., 2008; Sherkat et al., 2010). Positive attitudes toward SSM are linked to younger rather than older people (Brumbaugh et al., 2008). This may be explained by the socialization of young generations who have been more exposed to diverse models of sex, love, family, and culture than older generations who were educated with traditional models of marriage and parenting (Hegarty, 2018). Furthermore, heterosexual men often have more negative attitudes toward SSM and LGP compared to women, presumably because of their acculturation to more rigid sexual and gender roles and expectations based on gender role traditionalism, heterosexism, and masculine ideals regarding virility, strength, and male dominance (Costa et al., 2017; Vecho et al., 2018). Men's hostile attitudes are also stronger against gay males than lesbian couples; these are primarily based on heterosexism and traditional gender role beliefs (Costa et al., 2017; Salvati, Ioverno, Giacomantonio, \& Baiocco, 2016). By contrast, women tend to have a more fluid vision of gender and sexuality, as well as a more flexible and inclusive view of family relationships (Wills \& Crawford, 2000).

Among the psychological predictors of attitudes toward LG people, SSM, and LGP, intergroup contact with LG people (a frequency of interaction with lesbian and gay people) has been found to be one of the most relevant (Gross, Vecho, Gratton, D'Amore, \& Green, 2018; Vecho et al., 2018). Intergroup contact tends to decrease prejudice, especially if this contact entails a positive interaction between majority and minority group members (Pettigrew \& Tropp, 2008; Vezzali \& Stathi, 2017). Herek (2002) found that those without contact with LG people have more hostile attitudes toward them. As sexual prejudice is an antecedent of attitudes toward SSM and LGP (Herek, 2002), personal contact with LG people strongly predicts support for SSM as well (Herek \& Capitanio, 1996). Those with more contact with lesbian, gay, bisexual, and transgender (LGBT) people and those with LGBT friends and/or family members have more positive attitudes toward LG people, SSM, and LGP than those without LGBT people in their social networks (Costa et al., 2015; Merino, 2013). Furthermore, contact satisfaction (the degree of fulfillment in the interaction with LG people) is an important variable explaining positive attitudes toward minority groups. In fact, contact satisfaction fosters empathy and reduces anxiety (Vezzali, Capozza, Mari, \& Hichy, 2007; Vezzali, Turner, Capozza, \& Trifiletti, 2018).

Gender role traditionalism is also an important predictor of attitudes toward LG people, SSM, and LGP. This concept refers to a set of beliefs regarding the appropriate and expected behaviors for men and women based on traditional gender role behaviors. For instance, women traditionally are expected to be dependent, submissive, child oriented, family oriented, and emotionally expressive, whereas men are expected to be autonomous, strong, dominant, career oriented, and stoic (Webb, Chonody, \& Kavanagh, 2017). Gender role traditionalism appears to be associated with negative attitudes toward LG parenting because SSM and LGP break traditional gender role expectations (Pacilli, Taurino, Jost, \& van der Toorn, 2011).

Another important predictor of negative attitudes toward LG people, SSM, and LGP is religious involvement (Whitehead, 2018). Sherkat et al. (2010) found that greater religiosity and frequency of attending religious services are among the strongest correlates of these negative attitudes toward LG people. In many religions, coupling and parenting are prescribed as heterosexual activities, and same-sex orientation is viewed as inherently sinful. This norm is reinforced by the secular transmission of sexual and gender role prescriptions (Sherkat et al., 2010). In this context, it is not surprising to see that religiosity is strongly associated with the approval of traditional gender roles in most European countries. Moreover, negative attitudes toward LG people and samesex relationships are prevalent in the more conservative, fundamentalist branches of different religious denominations (Whitley, 2009). Importantly, it has to be noted that many other religious denominations, such as mainstream Protestantism, tend to have a more accepting stance on same-sex couples and sexuality (Barringer \& Gay, 2016).

Considering the aforementioned predictors of attitudes toward SSM and LGP, we were interested in the relationship between the sociopolitical context - especially the legal status of SSM and LGP - and support for SSM and LGP. Research has suggested that negative attitudes toward minority groups strongly depend on the perceived normative appropriateness of such attitudes within one's group or community (see Crandall, Eshelman, \& O'Brien, 2002). This is based on the assumption that people use their groups as a reference for defining their own views, especially in the face of uncertainty 
(e.g., Sherif \& Sherif, 1964). Research has demonstrated the importance of normative influences on attitudes toward LG people (Pereira, Monteiro, \& Camino, 2009). Thus, laws represent strong cultural norms that may be associated with various attitudes between groups within a society, i.e., between the heterosexual majority and LG people (including the legal statuses extended to the latter). We, therefore, explored the positive and negative social attitudes toward these issues in countries where SSM and LGP have varying legal statuses.

\section{Aims and Hypotheses}

We evaluated the effects of country- and individual-level factors on heterosexuals' attitudes toward SSM and LGP. We also investigated whether the impact of country-level variables on support for SSM and LGP was mediated ${ }^{1}$ by psychological variables. Our hypotheses were as follows:

H1. Participants from countries with more conservative legislations (i.e., Poland and Greece) have more negative attitudes toward LG people and lower levels of support for SSM and LGP than participants from countries with more progressive legislation (i.e., Belgium, France, Italy, Portugal, and Spain).

H2. Psychological variables are associated with attitudes toward LG people and support for SSM and LGP. More specifically, greater religiosity and gender role traditionalism are associated with more negative attitudes toward LG people and with weaker support for SSM and LGP, whereas female gender and greater contact with LG people are associated with more positive attitudes toward LG people and greater support for SSM and LGP. For this hypothesis, we developed a theoretical model that was empirically tested (see Fig. 2).

H3. Cultural differences are mediated by religiosity, gender role traditionalism, and contact with LG people. These three psychological variables, which were based on our examination of various countries, indirectly affect the levels of support (Poland and Greece $=0$, others (Italy, Portugal, France, Belgium, and Spain) $=1$ ) for SSM and LGP. Furthermore, we postulated that the effects of these variables are mediated by negative attitudes toward LG people.

\section{Method}

\section{Participants and Procedures}

We conducted a large-scale study from April 2012 to November 2014. A total of 13,403 heterosexual participants

\footnotetext{
${ }^{1}$ A mediation means that the effect of a predictor on an outcome (dependent variable) is explained either in whole or in part (partial mediation) by a third variable (see Baron \& Kenny, 1986) called the mediator.
}

completed an online survey about their attitudes toward SSM and LGP. The number of participants per country was as follows: Belgium $(n=4628)$, France $(n=2267)$, Greece $(n=293)$, Italy $(n=3833)$, Poland $(n=644)$, Portugal $(n=1106)$, and Spain $(n=632)$. The survey was presented in the participants' native language. The participants $\left(M_{\text {age }}=24.65, S D=7.63\right)$ were university students, a third of whom were enrolled in social science faculties (Table 2). They were recruited online across different European universities. We emailed the research description, SurveyMonkey link, and inclusion criteria to the universities and requested dissemination via email to the enrolled students. The students were informed that participation was voluntary and anonymous, and they were asked for their consent to participate. All procedures followed the ethical standards of the relevant institutional and/or national research committees.

\section{Measures}

The administered instruments were taken from previous studies and back translated to ensure construct equivalence (Brislin, 1970). The English version of the questionnaire, full data, an executable R Markdown file including all the code used to generate these analyses, and the output of this code are available on the online supplementary material (https://osf.io/jnq9h/).

Religiosity We assessed religiosity using the following two items: "How often do you attend religious services?" $(1=$ never to $6=$ once a week or more) and "How important is religion for you?" ( $1=$ not at all important to $5=$ very important). As both items were strongly correlated $(r=.69)$, we transformed the latter item on a scale ranging from 1 to 6 and then averaged both items to form a single scale so that higher scores indicated a higher level of religiosity.

Gender Role Traditionalism We measured attitudes toward gender role traditionalism using the short version of the FemMasc Scale (Honegger, Nabavi, \& Green, 2005), which assesses the approval of traditional gender roles (e.g., "A woman should be more family oriented than career oriented," and "A man should be the head of the family") on a sevenpoint Likert scale $(1=$ strongly disagree to $6=$ strongly agree). Higher scores indicated greater endorsement of traditional gender roles.

Contact with LG People We measured contact with LG people using the following three questions: "How many lesbian and/ or gay family members (including immediate and extended family members) do you have?", "How many lesbian and/or gay friends do you have?", and "How many lesbian and/or gay coworkers or acquaintances in the community do you have?" We calculated the contact score by adding the number 


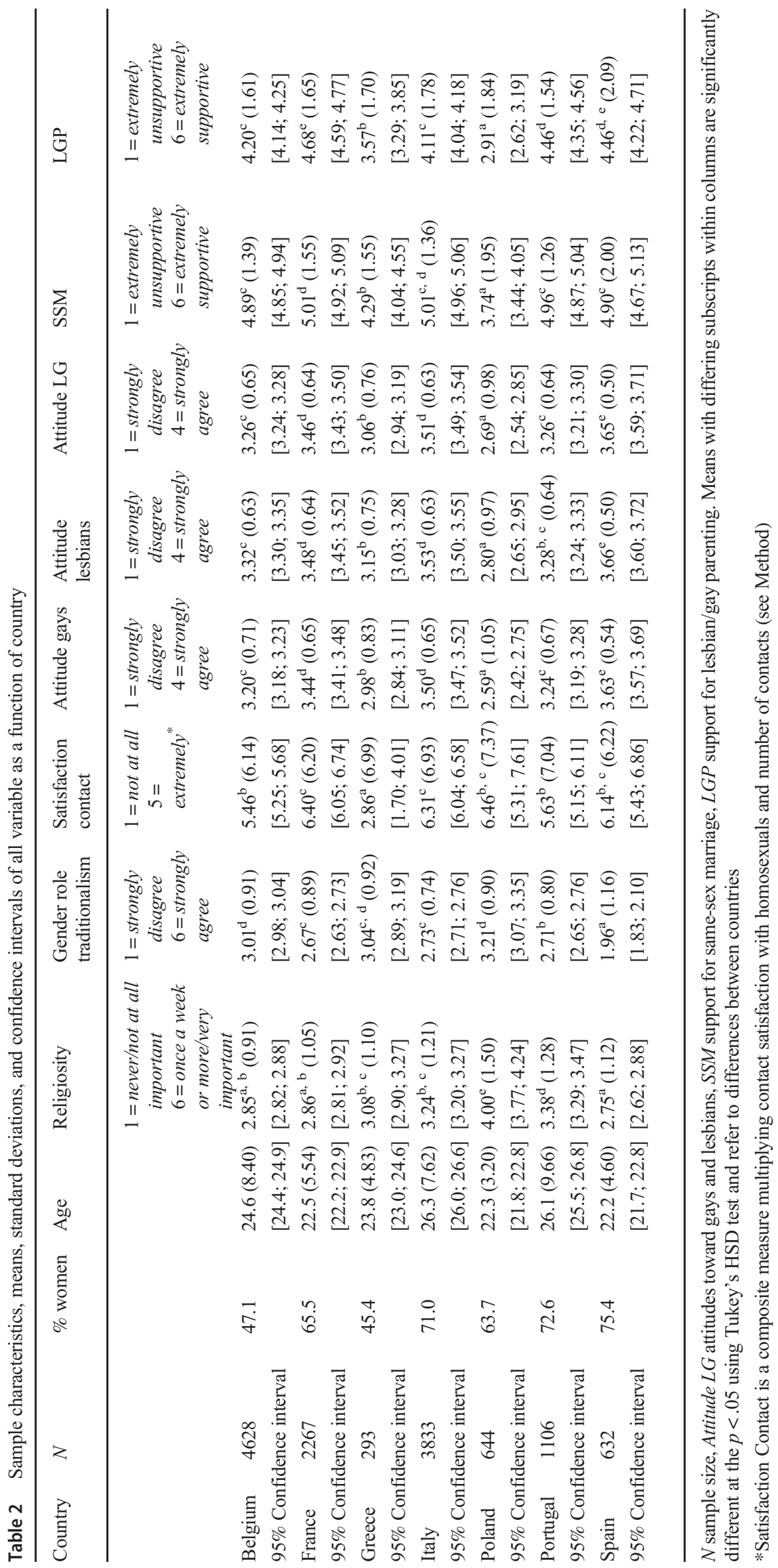


of contacts in each category. ${ }^{2}$ We then measured the perceived contact satisfaction using the following item for the three groups (family members, friends, and coworkers): "How satisfied are you with your relationship with this/these person(s)?" on a five-point Likert format (from $1=$ not at all to $5=$ extremely). We calculated the contact satisfaction score using the average satisfaction score for friends and coworkers, respectively, with $r=.50$ (because the family item correlated negatively with the other items for Greek participants). Our main indicator was an aggregated variable multiplying the number of contacts by the quality of contact (contact satisfaction). Before doing so, we removed 3 from the raw value. Given that the scale ranged between 1 and 5, negative scores indicated poor contact quality, and positive scores indicated good contact. This aggregated variable is henceforth labeled "contact with LG people."

Attitudes Toward LG People We measured the participants' attitudes toward LG people using the Attitudes toward Lesbians and Gay Men Scale (Herek, 1984). This scale is composed of two sets of five items measuring attitudes toward LG people separately (e.g., "Sex between two men/women is just plain wrong" [reversed item], and "Male/female homosexuality is merely a different kind of lifestyle that should not be condemned") on a five-point Likert scale ( $1=$ strongly disagree to $5=$ strongly agree). Given the high correlations between both attitudes (.90 for the whole sample), we calculated the mean score with a higher score-indicating more positive attitudes toward LG people.

Support for SSM We assessed the level of support for SSM using the last item of Katuzny and Green's Same-Sex Marriage Scale (2013), "How supportive or unsupportive are you of same-sex marriage now?", which was measured on a six-point Likert scale $(1=$ extremely unsupportive to $6=$ extremely supportive $)$. Higher scores reflect higher levels of support for SSM.

Support for LGP We assessed LGP using a single item from D'Amore and Green's Same-Sex Parenting Scale (2012): "How supportive or unsupportive are you of lesbian and gay parenting now?", which was measured on a six-point Likert scale $(1=$ extremely unsupportive to $6=$ extremely supportive $)$. Higher scores reflect elevated levels of support for LGP.

\section{Multigroup Measurement Invariance}

We tested whether all participants interpreted the survey questions in a similar manner using a multigroup measurement

\footnotetext{
$\overline{2}$ The main analyses were conducted with this aggregated contact score. The same analyses were run using the three contact categories in place of the aggregated indicators. The results of these analyses were similar to those of the former.
}

invariance analysis (Byrne, 2004). We estimated different levels of invariance for each measurement. Model fit was assessed using multiple fit indices (Tanaka, 1993). The Root Mean Square Error of Approximation (RMSEA) should be $\leq .08$, and the Comparative Fit Index (CFI) should be $\geq .95$ (see Byrne, 2004; Capozza, Vezzali, Trifiletti, \& Falvo, 2010; Hu \& Bentler, 1999). First, we tested the unconstrained (i.e., configural) model to verify that the general factor structure of the measurement was the same across different groups. To determine whether measurement invariance was present, we tested metric invariance by comparing the metric model with constrained measurement weights to the unconstrained configural model. Then, we tested scalar invariance by comparing the scalar model with constrained intercepts to the metric model with constrained measurement weights. Changes in $\mathrm{CFI}<.01$ and RMSEA of $<.015$ are considered indications of non-invariance (Chen, 2007).

The initial model included six latent variables with the last three (LGP, SSM, and contact with LG people) having only a single indicator. This model had a poor fit $\left(\chi^{2}[263]=14,510\right.$, $p=0, \mathrm{CFI}=.75, \mathrm{RMSEA}=.11)$. In an attempt to improve the fit of the model, we inspected factor loadings among each culture and removed six items ${ }^{3}$ that loaded too weakly $(<.40)$ on their latent constructs. Additionally, we examined the correlations between the residuals of items loading on the same scales (i.e., either attitudes toward gay and lesbians or gender role traditionalism). When they were sufficiently high ( $>.25$ in absolute values), we included the relevant covariances between the disturbances in the model (see the Online Supplementary Material, Section 8.1.2, for the full adapted model). As a result of these changes, the model had a much better fit $\left(\chi^{2}[255]=3825.33, p=0, \mathrm{CFI}=0.94\right.$, RMSEA $=$ 0.06 ).

We first tested measurement invariance using a configural model including all variables, which revealed a good model fit $\left(\chi^{2}=6609.548, \mathrm{df}=1785, \mathrm{CFI}=.921, \mathrm{RMSEA}=.065\right)$; then, we compared this configural model with a constrained model $\left(\chi^{2}=7370.455, \mathrm{df}=1899, \mathrm{CFI}=.910, \mathrm{RMSEA}=.067\right)$. This confirmed that the full metric invariance was achieved $(\triangle \mathrm{CFI}=.011, \Delta \mathrm{RMSEA}=.002)$. Finally, we created an additional constrained scalar model with constrained intercepts and loadings $\left(\chi^{2}=9816.256, \mathrm{df}=2013, \mathrm{CFI}=.872\right.$, RMSEA $=.078)$ and compared it with the metric model with constrained measurement weights $(\triangle \mathrm{CFI}=.038$, $\triangle$ RMSEA $=.011$ ). As expected, the significant changes in CFI indicated that scalar invariance was not achieved, suggesting that the model varies across cultures; we, therefore, focused on country-specific effects.

Ultimately, all standardized factor loadings were significant and above the conventional threshold $(\geq .40)$, confirming

\footnotetext{
${ }^{3}$ Gender role traditionalism: items 5, 7, 9, and 12 from the original scale. Attitude toward LG people: item 5 (see online supplementary material).
} 
that all items exhibited sufficient loadings across all cultures. Specifically, Cronbach alphas were good for gender role traditionalism $($ Belgium $=.90 ;$ France $=.90 ;$ Greece $=.90$; Italy $=.89$; Poland $=.89$; Portugal $=.87$; Spain $=.94)$, attitudes toward gays $($ Belgium $=.82 ;$ France $=.81$; Greece $=.87 ;$ Italy $=.80 ;$ Poland $=.89 ;$ Portugal $=.84 ;$ Spain $=.72)$, attitudes toward lesbians $($ Belgium $=.79$; France $=.82 ;$ Greece $=.86 ;$ Italy $=.79 ;$ Poland $=.87$; Portugal $=.84$; Spain $=.69$ ), and attitudes toward LG people combined $($ Belgium $=.90 ;$ France $=.91 ;$ Greece $=.93$; Italy $=.90 ;$ Poland $=.94 ;$ Portugal $=.93 ;$ Spain $=.69) .{ }^{4}$

\section{Results}

\section{Differences in Psychological Variables per Country $(\mathrm{H} 1)^{5}$}

Table 2 reports the sample characteristics, means, standard deviations, and confidence intervals on the measured variables as a function of country. We first used a comparative approach at the country level. For H1, we tested whether the participants from countries with more conservative legislation had lower levels of support for SSM and LGP than their counterpart neighbors. We examined the means of the psychological variables as a function of country by using ANOVA followed by post hoc comparisons (using Tukey's HSD). We included participants who had no missing values on the relevant variables for each analysis, meaning that the sample size can differ across analyses. In all cases, the null hypothesis of the ANOVAs could be safely rejected: $F(6,12,462)=78.1$, $p<.001, \eta_{\mathrm{p}}{ }^{2}=.036$ for SSM; $F(6,12,217)=99.5, p<.001$, $\eta_{\mathrm{p}}{ }^{2}=.047$ for LGP; $F(6,11,633)=208, p<.001, \eta_{\mathrm{p}}{ }^{2}=.097$ for attitudes toward gay men; $F(6,11,619)=148, p<.001$, $\eta_{\mathrm{p}}{ }^{2}=.071$ for attitudes toward lesbian women; $F(6$, $11,398)=186, p<.001, \eta_{\mathrm{p}}{ }^{2}=.089$ for attitudes toward LG people combined; $F(6,12,591)=151, p<.001, \eta_{\mathrm{p}}^{2}=.067$ for religiosity; $F(6,10,630)=165, p<.001, \eta_{\mathrm{p}}{ }^{2}=.085$ for gender role traditionalism; and $F(6,5655)=6.47, p<.001$, $\eta_{\mathrm{p}}{ }^{2}=.001$ for contact with LG people. ${ }^{6}$ These post hoc tests are reported in Table 2 and allow for differentiating various differences between countries. Tukey's HSD tests showed that support for SSM and LGP in Poland and, to a lesser extent, in Greece was significantly lower than that in other

\footnotetext{
${ }^{4}$ Because many participants seem to have no contact with LG people at all, we calculated alphas as a function of missing values (i.e., without the item "family").

${ }^{5}$ We relied on the pairwise deletion of missing values for ANOVAs and correlations and on the listwise deletion of missing values for mixed models and structural equation modeling (for detailed information and differences between both approaches in our dataset, see online supplementary materials). ${ }^{6}$ Due to slight differences in the age and gender composition of the samples, we reproduced these analyses using ANCOVAs with gender and age as covariates. This had no bearing on the results.
}

countries (see Fig. 1). In all countries, support for SSM is higher than support for LGP. Poland, followed by Greece, had more negative attitudes toward LG people than the other countries (see Table 2). These results provided empirical support for $\mathrm{H} 1$.

\section{Psychological Variables Associated with Support for SSM and LGP (H2)}

For $\mathrm{H} 2$, we investigated the psychological variables associated with support for SSM and LGP. The correlations between latent variables are reported in Table 3 . The findings showed a significant negative correlation between religiosity and support for SSM and LGP. Correlations between gender role traditionalism and support for SSM and LGP followed the same trend. The higher the level of gender role traditionalism, the lower the levels of support for SSM and LGP. By contrast, the contact with LG people score was positively correlated with support for SSM and LGP. Attitude toward LG people was also positively correlated with support for SSM and LGP. In summary, $\mathrm{H} 2$ received empirical support.

Mixed Models The goal of these analyses was to examine the effects of each of our psychological variables on attitudes toward SSM and LGP. To investigate this possibility, we used a mixed model, treating country as a random level 2 factor and the participants as a level 1 factor. We relied on the packages LME4 (Bates, 2005) and lmerTest (Kuznetsova, Brockhoff, \& Christensen, 2017). All predictors were previously centered across the whole sample. ${ }^{7}$

For these and the following analyses, we only used complete cases (i.e., listwise deletion, $n=4265$ for LGP and $n=$ 4227 for SSM). Regarding attitudes toward LGP, we entered all psychological predictors simultaneously, letting the intercept vary randomly per country. Table 4 presents the estimations for the random portion of the model, as well as the standardized coefficients for the fixed portion. Attitudes toward LG people and contact with LG people significantly and positively predicted attitudes toward LGP, whereas religiosity had a negative effect. A negative effect was also found for gender role traditionalism and male gender, although the size of the coefficients was much smaller. Regarding attitudes toward SSM, the same pattern of effects emerged. Both gender and age significantly predicted attitudes toward SSM (see Table 4).

Hypothesized SEM Model Our next research questions involved examining whether the psychological predictors could

\footnotetext{
${ }^{7}$ We did not enter contextual level 2 variables (e.g., mean of each predictor within a country: cf. Enders and Tofighi, 2007) because of the insufficient number of level 2 observations (seven countries). The purpose of the analysis, therefore, is to not examine the difference between countries but the effect of each predictor, taking into account between-country differences.
} 
Fig. 1 Attitudes toward same-sex marriage and lesbian/gay parenting as a function of country

6-

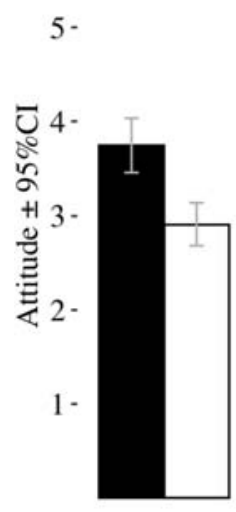

Poland

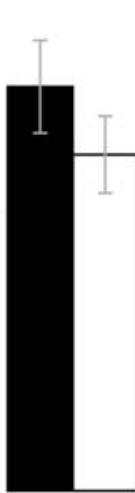

Greece

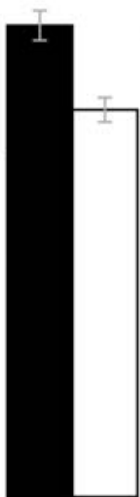

Italy

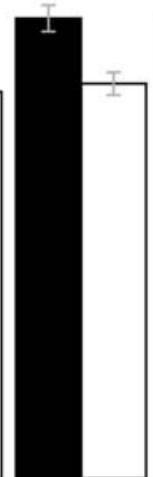

Belgium

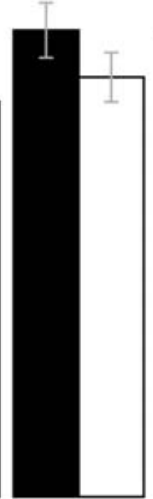

Portugal

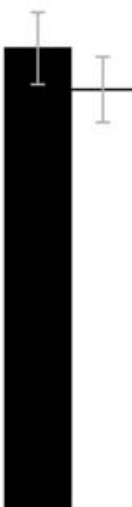

Spain

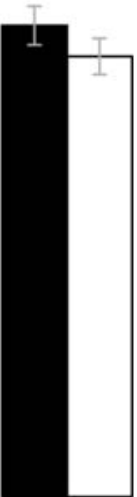

France account for between-country differences in attitudes toward SSM and LGP. An examination of the means (see Table 2) revealed that two countries, Poland and Greece, had clearly more negative attitudes than the others. We, therefore, created a dummy variable contrasting these two groups (Poland and Greece $=0$, others $=1$ ). To examine whether the differences in attitudes toward SSM and LGP between these two clusters was mediated by religiosity, contact with LG people, and attitudes toward LG people, we performed a structural equation modeling analysis using the Lavaan package (Rosseel, 2012) in R. Table 5 and Fig. 2 report the standardized path coefficients for all of these variables. The model had a satisfactory fit $\left(X^{2}[293]=4432.39, p=0, \mathrm{CFI}=0.93\right.$, $\left.\mathrm{RMSEA}=0.06\right)$. An examination of the coefficients suggested that country predicted religiosity and gender role traditionalism (but not contact with LG people), which predicted attitudes toward
LG people, whereas the latter predicted attitudes toward SSM and LGP. When the indirect effects are compared, it is the path through religiosity that most strongly explains the difference between countries (see Table 5). The effects of religiosity and gender role traditionalism were fully mediated for SSM and partially mediated for LGP by attitudes toward LG people, although these residual effects were only substantial for religiosity.

\section{Discussion}

We evaluated a theoretical model analyzing attitudes toward LG people, SSM, and LGP in a large European sample of heterosexual youths. Previous studies have mostly focused on
Table 3 Bivariate correlations for the whole sample of participants

\begin{tabular}{|c|c|c|c|c|c|c|c|c|c|}
\hline & 1 & 2 & 3 & 4 & 5 & 6 & 7 & 8 & 9 \\
\hline 1. Age & - & & & & & & & & \\
\hline 2. Support SSM & $-.02^{* *}$ & - & & & & & & & \\
\hline 3. Support LGP & -.01 & $.77^{* *}$ & - & & & & & & \\
\hline 4. Religiosity & $.05^{* *}$ & $-.41^{* *}$ & $-.38^{* *}$ & - & & & & & \\
\hline 5. Satisfaction contact & $.08^{* *}$ & $.26^{* *}$ & $.27^{* *}$ & $-.06^{* *}$ & - & & & & \\
\hline $\begin{array}{l}\text { 6. Gender role } \\
\text { traditionalism }\end{array}$ & $-.08^{* *}$ & $-.29^{* *}$ & $-.29^{* * *}$ & $.22^{* * *}$ & $-.15^{* *}$ & - & & & \\
\hline 7. Attitude gays & $.02^{*}$ & $.73^{* *}$ & $.65^{* *}$ & $-.35^{* *}$ & $.28^{* *}$ & $-.44^{* *}$ & - & & \\
\hline 8. Attitude lesbians & .01 & $.72^{* *}$ & $.64^{* *}$ & $-.39^{* *}$ & $.26^{* *}$ & $-.39^{* *}$ & $.90^{* *}$ & - & \\
\hline $\begin{array}{l}\text { 9. Attitudes gays and } \\
\text { lesbians }\end{array}$ & .02 & $.75^{* *}$ & $.66^{* *}$ & $-.38^{* * *}$ & $.28^{* *}$ & $-.43^{* *}$ & $.98^{* *}$ & $.97^{* *}$ & - \\
\hline
\end{tabular}


Table 4 Linear mixed model treating country as a random level 2 factor and participants as level 1

\begin{tabular}{lllllllll}
\hline Measures & Random effect country & Random effect residual & $\beta$ age & $\beta$ gender & $\beta$ religiosity & $\beta$ GRT & $\beta$ Att LG & $\beta$ contact \\
\hline Same-sex parenting & $.051(.225)$ & $1.275(1.129)$ & -.020 & $-.052^{* * *}$ & $-.179^{* * *}$ & $-.077^{* * *}$ & $.476^{* * *}$ & $.125^{* * *}$ \\
Same-sex marriage & $.014(.117)$ & $0.689(0.830)$ & $-.051^{* * *}$ & $-.070^{* * *}$ & $-.182^{* * *}$ & $-.059^{* * *}$ & $.555^{* * *}$ & $.100^{* * *}$ \\
\hline
\end{tabular}

$* * *=p<.001$

$\beta$ standardized coefficients beta of each psychological-level variable, GRT gender role traditionalism, Att LG attitudes toward gays and lesbians, Contact the quality X number of contacts score. We did not enter contact satisfaction, as several participants did not report contact. Values reported in Random effect columns correspond to variance and standard deviations. Number of countries $=7$

attitudes toward LG people, SSM, and LGP in a single country (Costa et al., 2017; Ioverno et al., 2018; Vecho et al., 2018), ${ }^{8}$ or two countries (Costa \& Salinas-Quiroz, 2019).

To our knowledge, only the European Commission study (2015) compared attitudes across different European countries. The present research contributes to the literature by considering the effects of country- and psychological-level variables on SSM and LGP.

We found that participants from countries with more conservative legislation (Poland and Greece) had lower levels of support for SSM and LGP than participants from countries with more progressive legislation. Prejudice, therefore, can be understood as an outcome of salient group norms (Crandall et al., 2002). For example, the lack of recognition of civil rights in Poland and Greece may influence attitudes toward SSM and LGP by making them less acceptable. Thus, the sociopolitical context, namely, the legal status of SSM and LGP, influences the attitudes toward such issues. These results confirm research findings across Europe (Commissioner for Human Rights, 2011; European Commission, 2015).

Gender role traditionalism also played an important role in predicting heterosexuals' attitudes toward SSM and LGP. Our study showed that traditional beliefs related to gender roles are highly predictive of negative attitudes toward LG people, SSM, and LGP (Whitley, 2009). Gender role traditionalism, followed by contact with LG people, differed little between countries and accounted for a much lower portion of the variance in attitudes. Greece and Poland differed much more from other countries in terms of higher gender role traditionalism and less contact satisfaction with LG people. Even among relatively comparable student samples, there seemed to be a sizable between-country variability in these factors.

The participants with higher religiosity showed more negative attitudes toward LG people and less support for SSM and LGP. These results also confirm previous findings (e.g., Denton, 2004). Many European religions remain reluctant to question the traditional representations of sexuality, gender,

\footnotetext{
${ }^{8}$ Data of these studies were collected between 2012 and 2014 in the framework of the present European Research on Attitudes toward Same-Sex Marriage and LG parenting coordinated by the first and the third author.
}

and family roles. Most of them oppose LGP not only because they consider same-sex relationships immoral and sinful but also because they are unable to replicate the heteronormative family model in holy writings. Thus, religiosity is a strong factor that shapes the representations of and attitudes toward SSM and LGP.

Religiosity and attitudes toward LG people were the strongest psychological predictors of attitudes toward SSM and LGP. The independent effect of religiosity from participants' level of sexual prejudice is intriguing. Highly religious participants are more likely to be exposed to negative and stigmatizing discourse from leaders. Beyond their own beliefs regarding LG people, fighting SSM and LGP may become a form of politicized identity for such individuals (Simon \& Klandermans, 2001). A possible explanation is that people who consider themselves religious would identify their religious community as part of their social group. Thus, if the religions that are dominant in Poland and Greece are more traditional in their doctrine, then this could potentially explain our findings that Poland and Greece were less positive in their attitudes toward LG, SSM, and SSP because of social norms. Regardless of the specific religion, the cultural and social importance attributed to religion, and the level of the country's secularism may further elucidate these findings. We can also speculate that Greek Orthodox Church in Greece and the Catholic Church in Poland actually "may be" more literal and conservative than the contemporary Catholic Church in Spain and France. Greater religiosity predicted more negative attitudes toward LG, which predicted additional negative attitudes toward SSM and SSP. Attitudes toward LG did not fully mediate the relationship; that means that more religious people hold negative attitudes toward SSM and SSP because they hold more negative attitudes toward LG. It is likely that many religious teachings emphasize heterosexual norms for people's identities, families, and marriages - creating a system of unacceptance.

Lastly, we found that religiosity and attitudes toward LG people (but not contact satisfaction with LG people) mediated the differences in attitudes toward SSM and LGP between countries.

Across the seven countries, participants who reported having more contact satisfaction with LG people displayed greater support for SSM and LGP, as well as more 
Table 5 Standardized path coefficients for all variables on SSM and LGP

\begin{tabular}{|c|c|c|c|c|c|c|}
\hline Measures & Direct effect $\beta$ & Direct effect $z$ & Direct effect CI & Indirect effect $\beta$ & Indirect effect $z$ & Indirect effect CI \\
\hline \multicolumn{7}{|l|}{ SSM } \\
\hline Religiosity & $-.202(.014)$ & $-14.312 * * *$ & {$[-.229 ;-.174]$} & $.020(.003)$ & $6.419 * * *$ & {$[.014 ; .027]$} \\
\hline Gender role traditionalism & $-.053(.014)$ & $-3.778 * * *$ & {$[-.080 ;-.025]$} & $.008(.002)$ & $3.505 * * *$ & {$[.004 ; .013]$} \\
\hline Satisfaction contact & $.080(.012)$ & $6.780 * * *$ & {$[.057 ; .103]$} & $.001(.002)$ & 0.585 & {$[-.002 ; .004]$} \\
\hline Attitudes gays lesbians & $.567(.014)$ & $41.941 * * *$ & {$[.540 ; .593]$} & - & - & - \\
\hline Gender & $-.052(.012)$ & $-4.486 * * *$ & {$[-.075 ;-.029]$} & - & - & - \\
\hline Country & $.001(.011)$ & 0.090 & {$[-.021 ; .023]$} & - & - & - \\
\hline \multicolumn{7}{|l|}{ LGP } \\
\hline Religiosity & $-.213(.015)$ & $-13.990 * * *$ & {$[-.243 ;-.183]$} & $.016(.003)$ & $6.344 * * *$ & {$[.011 ; .021]$} \\
\hline Gender role traditionalism & $-.091(.015)$ & $-6.018 * * *$ & {$[-.121 ;-.061]$} & $.007(.002)$ & $3.494 * * *$ & {$[.003 ; .010]$} \\
\hline Satisfaction contact & $.114(.013)$ & $8.797 * * *$ & {$[.089 ; .139]$} & $.001(.001)$ & 0.585 & {$[-.002 ; .003]$} \\
\hline Attitudes gays lesbians & $.445(.015)$ & $29.161 * * *$ & {$[.415 ; .475]$} & - & - & - \\
\hline Gender & $-.039(.013)$ & $-3.032 * * *$ & {$[-.064 ;-.014]$} & - & - & - \\
\hline Country & $.027(.012)$ & $2.162 *$ & {$[.003 ; .051]$} & - & - & - \\
\hline
\end{tabular}

$* * * p<.001$

$* p=.035$

$\beta$ standardized coefficients, $C I 95 \%$ confidence intervals. Gender $($ women $=0$, men $=1)$. Country $($ Poland and Greece $=0$, others $=1)$

accepting attitudes toward LG people. This is consistent with previous findings revealing that heterosexual individuals who had greater contact with sexual minorities showed more positive attitudes toward LG's people civil rights (see Collier, Bos, \& Sandfort, 2012; Herek, 1984).
We found that contact with LG people was positively associated with support for SSM and LGP. These findings suggest that sexual prejudice can be reduced if efforts to promote contact with LG individuals, couples, and families are made in different sectors of society.

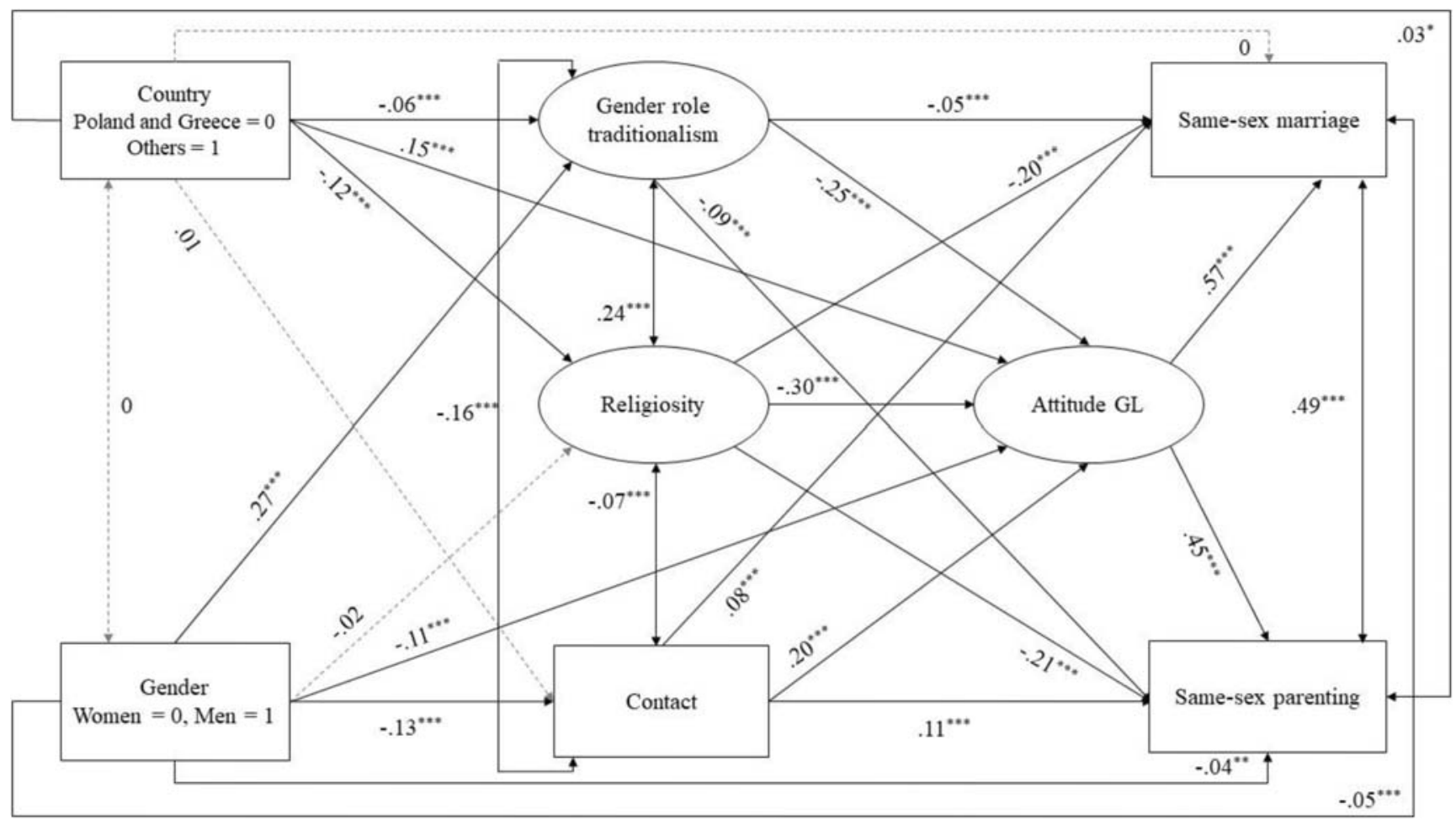

Fig. 2 Structural Equation Model 
Similar to other studies (e.g.,Costa et al., 2017; Vecho et al., 2018), our work revealed that attitudes toward LG people were also positively associated with support for SSM and LGP and were a direct predictor of LGP and SSM. Furthermore, they mediated the link between other psychological variables and support for SSM and LGP.

\section{Limitations}

The first limitation of this study is the composition of the sample. The study sample encompassed university students who are more educated and have potentially more contact with other cultures compared with the general population (Henrich, Heine, $\&$ Norenzayan, 2010). These students were not randomly selected from all colleges/universities in each country, so the sample is not representative of the general population.

A second limitation point to consider is that our data was collected between 2012 and 2014. Since 2014, France and Portugal have approved same-sex marriage and adoption. In Italy, same-sex registered partnership was approved. These legal advancements may have strengthened the trends that we found for these countries although social change takes a long time to become noticeable and do not necessarily accompany legislative changes.

The third limitation is the type of measurement used to assess our main variables. Because of the length of the questionnaire, the measures were often limited to one or a few items. This raises questions about the reliability of our indicators, especially the measures of SSM and LGP. Although these measures were used in previous studies (Costa et al., 2017; Vecho et al., 2018), the absence of multi-item measures remains a major limitation. Furthermore, the use of a single item to appraise attitudes toward LGP might have reduced the complexity of the concept, as these attitudes might differ according to the gender of lesbian/gay parents. Although we studied general attitudes toward SSM and LGP, different variations could exist in attitudes toward the different paths to parenthood, such as adoption, foster care, and surrogacy.

Fourth, in the present study, we excluded a large number of subjects who displayed missing values on at least one of our core variables. This raises the possibility that the excluded participants differed from the rest of the sample and that our analyses are therefore not representative of the overall population. Specifically, if excluded participants are more conservative than the rest of the sample, our analyses may suffer from a restricted range on some variables. To address this possibility, we examined scores on our core variables among included and excluded participants. The mean differences between the two groups were small (all $|\mathrm{ds}|<.15$ ) and did not point consistently in one direction (e.g., excluded participants tended to be slightly less favorable toward gay marriage but slightly more favorable to gender traditionalism), which is inconsistent with an overall "liberal bias" among the participants that were kept in the analyses. Similarly, the interquartile range on all variables was largely similar in both groups. Nonetheless, further studies may consider using more sophisticated missing values replacement methods and, even more importantly, seek to minimize them.

Fifth, the sample sizes vary across countries. This has two important implications: the estimations of the relations between variables are largely determined by countries with greater sample sizes (e.g., Belgium, Italy) than those with smaller sample sizes. Specifically, as the sample size increases, the confidence in the estimate and model fit statistics increases, and the uncertainty decreases which lead to greater precision. Second, and in the same vein, the estimation of the true value of the mean in the population is more precise in the more heavily represented countries, but as indicated in Table 2, the confidence intervals remain small even in Greece (the smallest sample). We encourage scholars to rely on large and close sample sizes when using SEM in the context of cross-national research to more accurately capture national differences.

Sixth, although measurement invariance analysis ensured that metric invariance was established, scalar invariance was not, showing that the relations between variables differ across groups. Note however that all standardized factor loadings were significant and above the conventional threshold confirming that all items exhibited sufficient loadings across all cultures, which translates into Cronbach alphas ranging from satisfactory to excellent for each country. This expected level of (scalar) invariance suggests that there are larger forces such as cultural norms or developmental differences influencing the way that participants developed attitudes toward SSM and SSP. Examining cultural differences is a major strength of this work, but the nuanced intricacies specific to the crosscultural samples added limitations. Consequently, future work should systematically rely on measurement invariance analysis and aim at testing for multiple-group factor analysis alignment to estimate group-specific factor means and variances without requiring exact measurement invariance (Asparouhov \& Muthén, 2014).

The seventh limitation is concerned with the crosssectional design of the study, which does not allow for the determination of causality. Future studies should use longitudinal designs to better assess attitudes toward SSM and LGP and their changes over time. We hope to follow-up our participants for this purpose.

\section{Conclusions}

Attitudes toward same-sex couples and parents in Europe vary across geographical areas based on sociopolitical contexts. All seven countries show majority acceptance of LGBT people 
and specifically show majority support among college students for same-sex marriage and lesbian/gay parenting.

Heterosexuals' greater exposure to LG people and greater satisfaction in relationships with LG people is associated with greater acceptance of LGs, support for same-sex marriage, and support for LG parenting. To increase heterosexuals' support of LGs equal rights, community institutions (secondary schools, colleges/universities, religious communities) should create more opportunities for heterosexuals to interact with openly LG people and have positive experiences with them.

Future cross-country comparisons on SSM and LGP can be made to assess perceived norms. Research on attitudes toward SSM and LGP needs to be constantly updated because overall public attitudes, particularly young people's attitudes, tend to improve over time.

We hope that this study will motivate researchers and policymakers to consider the importance of the legal context in fostering respectful attitudes toward those who choose to engage in nontraditional family and parenting practices. Although the law is partially an outcome of such attitudes, we believe that social attitudes and laws are best viewed as having involved mutually reinforcing causals relationships regarding SSM and LGP.

Supplementary Information The online version contains supplementary material available at https://doi.org/10.1007/s13178-020-00511-4.

Acknowledgments We would like to thank all national researchers that participated in this study: Martine Gross and Emmanuel Gratton (France), Salvatore Ioverno and Vittorio Lingiardi (Italy), and Marta Dora (Poland).

\section{References}

Asparouhov, T., \& Muthén, B. (2014). Multiple-group factor analysis alignment. Structural Equation Modeling: A Multidisciplinary Journal, 21(4), 495-508 (also known as Webnote 18, version 3) http:/www.statmodel.com/examples/webnotes/webnote18_3.pdf.

Averett, P. E., \& Hedge, A. (2012). School social work and early childhood student's attitudes toward gay and lesbian families. Teaching in Higher Education, 17(5), 537-549. https://doi.org/10.1080/ 13562517.2012.658564.

Baiocco, R., Carone, N., Ioverno, S., \& Lingiardi, V. (2018). Same-sex and different-sex parent families in Italy: Is parents' sexual orientation associated with child health outcomes and parental dimensions? Journal of Developmental \& Behavioral Pediatrics, 39(7), 555563.

Baron, R. M., \& Kenny, D. A. (1986). The moderator-mediator variable distinction in social psychological research: Conceptual, strategic, and statistical considerations. Journal of Personality and Social Psychology, 51(6), 1173-1182.
Barringer, M. N., \& Gay, D. A. (2016). Happily religious: The surprising sources of happiness among lesbian, gay, bisexual, and transgender adults. Sociological Inquiry, 87(1), 75-96. https://doi.org/10.1111/ soin. 12154.

Bates, D. (2005). Fitting linear mixed models in R. R News, 5(1), 27-30.

Brislin, R. W. (1970). Back-translation for cross-cultural research. Journal of Cross-Cultural Psychology, 1, 185-216. https://doi.org/ 10.1177/135910457000100301.

Brumbaugh, S. M., Sanchez, L. A., Nock, S. L., \& Wright, J. D. (2008). Attitudes towards gay marriage in states undergoing marriage law transformation. Journal of Marriage and Family, 70(2), 345-359. https://doi.org/10.1111/j.1741-3737.2008.00486.x.

Byrne, B. M. (2004). Testing for multigroup invariance using AMOS graphics: A road less traveled. Structural Equation Modeling, 11, 272-300. https://doi.org/10.1207/s15328007sem1102_8.

Capozza, D., Vezzali, L., Trifiletti, E., Falvo, R., \& Favara, I. (2010). Improving intergroup relationships within and outside the contact situation: The role of common ingroup identity and emotions of empathy and anxiety. Testing, Psychometrics, Methodology in Applied Psychology, 17, 17-36.

Caroll, A., \& Mendos, L. R. (2017). State-sponsored homophobia. A world survey of sexual orientation law: Criminalization, protection and recognition (12th ed.). Retrieved from https://www.ilga.org/ downloads/2017/ILGA_State_Sponsored_Homophobia_2017_ WEB.pdf.

Carone, N., Baiocco, R., Ioverno, S., Chirumbolo, A., \& Lingiardi, V. (2017). Same-sex parent families in Italy: Validation of the coparenting scale-revised for lesbian mothers and gay fathers. European Journal of Developmental Psychology, 14(3), 367-379.

Carone, N., Lingiardi, V., Chirumbolo, A., \& Baiocco, R. (2018). Italian gay father families formed by surrogacy: Parenting, stigmatization, and children's psychological adjustment. Developmental Psychology, 54(10), 1904-1916.

Chen, F. F. (2007). Sensitivity of goodness of fit indexes to lack of measurement invariance. Structural Equation Modeling: $A$ Multidisciplinary Journal, 14(3), 464-504. https://doi.org/10.1080/ 10705510701301834

Clarke, V., Kitzinger, C., \& Potter, J. (2004). Kids are just cruel anyway: Lesbian and gay parents talk about homophobic bullying. British Journal of Social Psychology, 43(4), 531-550. https://doi.org/10. 1348/0144666042565362.

Collier, K. L., Bos, H. M. W., \& Sandfort, T. G. M. (2012). Intergroup contact, attitudes toward homosexuality, and the role of acceptance of gender non-conformity in young adolescents. Journal of Adolescence, 35(4), 899-907. https://doi.org/10.1016/j. adolescence.2011.12.010.

Commissioner for Human Rights. (2011). Discrimination on grounds of sexual orientation and gender identity in Europe. Strasbourg, France: Council of Europe.

Costa, P. A., Carneiro, F., Esposito, F., D’Amore, S., \& Green, R.-J. (2017). Sexual prejudice in Portugal: Results from the first wave European study on heterosexuals' attitudes toward gay and lesbian marriage and parenting. Sexuality Research and Social Policy, 52, 35-55. https://doi.org/10.1007/s13178-017-0292-y.

Costa, P. A., \& Davies, M. (2012). Portuguese adolescents' attitudes toward sexual minorities: Transphobia, homophobia, and gender role beliefs. Journal of Homosexuality, 59(10), 1424-1442. https:// doi.org/10.1080/00918369.2012.724944.

Costa, P. A., Pereira, H., \& Leal, I. (2015). "The contact hypothesis" and attitudes toward same-sex parenting. Sexuality Research and Social Policy, 12(2), 125-136. https://doi.org/10.1007/s13178-014-01718.

Costa, P. A., \& Salinas-Quiroz, F. (2019). A comparative study of attitudes toward same-gender parenting and gay and lesbian rights in 
Portugal and in Mexico. Journal of Homosexuality, 66(13), 19091926.

Crandall, C. S., Eshelman, A., \& O'Brien, L. (2002). Social norms and the expression and suppression of prejudice: The struggle for internalization. Journal of Personality and Social Psychology, 82(3), 359-378. https://doi.org/10.1037//0022-3514.82.3.359.

D'Amore, S., \& Green, R.-J. (2012). The D'Amore and Green Same-Sex Parenting Scale. Belgium: University of Liège.

Denton, M. L. (2004). Gender and marital decision making: Negotiating religious ideology and practice. Social Forces, 82(3), 1151-1180. https://doi.org/10.1353/sof.2004.0034.

Enders, C. K., \& Tofighi, D. (2007). Centering predictor variables in cross-sectional multilevel models: A new look at an old issue. Psychological Methods, 12(2), 121-138.

European Commission. (2015). Eurobarometer on Discrimination. Retrieved from https://ec.europa.eu/info/sites/info/files/factsheet eurobarometer_fundamental_rights_2015_en.pdf.

Farr, R. H. (2017). Does parental sexual orientation matter? A longitudinal follow-up of adoptive families with school-age children. Developmental Psychology, 53(2), 252-264.

Fedewa, A. L., Black, W. W., \& Ahn, S. (2015). Children and adolescents with same-gender parents: A meta-analytic approach in assessing outcomes. Journal of GLBT Family Studies, 11(1), 1-34. https:// doi.org/10.1080/1550428X.2013.869486.

Gartrell, N., Bos, H., \& Koh, A. (2018). National longitudinal lesbian family study - Mental health of adult offspring. The New England Journal of Medicine, 379(3), 297-299.

Green, R. J., Rubio, R. J., Rothblum, E. D., Bergman, K., \& Katuzny, K. E. (2019). Gay fathers by surrogacy: Prejudice, parenting, and wellbeing of female and male children. Psychology of Sexual Orientation and Gender Diversity, 6(3), 269-283.

Gross, M., Vecho, O., Gratton, E., D'Amore, S., \& Green, R.-J. (2018). Religious affiliation, religiosity, and attitudes toward same-sex parenting. Journal of GLBT Family Studies, 14(3), 238-259. https:// doi.org/10.1080/1550428X.2017.1326016.

Hegarty, P. (2018). A recent history of lesbian and gay psychology: From homophobia to LGBT. Oxon, England: Routledge.

Henrich, J., Heine, S., \& Norenzayan, A. (2010). The weirdest people in the world? Behavioral and Brain Sciences, 33(2-3), 61-83. https:// doi.org/10.1017/S0140525X0999152X.

Herek, G. M. (1984). Attitudes toward lesbians and gay men: A factor analytic study. Journal of Homosexuality, 10(1-2), 39-51. https:// doi.org/10.1300/J082v10n01 03.

Herek, G. M. (2002). Heterosexuals' attitudes toward bisexual men and women in the United States. The Journal of Sex Research, 39(4), 264-274. https://doi.org/10.1080/00224490209552150.

Herek, G. M., \& Capitanio, J. P. (1996). "Some of my best friends" intergroup contact, concealable stigma, and heterosexuals' attitudes toward gay men and lesbians. Personality and Social Psychology Bulletin, 22(4), 412-424. https://doi.org/10.1177/ 0146167296224007

Honegger, A. T., Nabavi, R., \& Green, R.-J. (2005). Femininity-masculinity attitudes, stress and conformity questionnaire. San Francisco, CA: California School of Professional Psychology.

Hooghe, M., \& Meeusen, C. (2013). Is same-sex marriage legislation related to attitudes toward homosexuality? Sexuality Research and Social Policy, 10(4), 258-268. https://doi.org/10.1007/s13178-0130125-6.

Hu, L. T., \& Bentler, P. M. (1999). Cutoff criteria for fit indexes in covariance structure analysis: Conventional criteria versus new alternatives. Structural Equation Modeling: A Multidisciplinary Journal, 6(1), 1-55.
Hull, K. E. (2003). The cultural power of law and the cultural enactment of legality: The case of same-sex marriage. Law and Social Inquiry, 28(3), 629-657.

Ioverno, S., Baiocco, R., Lingiardi, V., Verrastro, V., D’Amore, S., \& Green, R.-J. (2018). Attitudes towards same-sex parenting in Italy: The influence of traditional gender ideology. Culture, Health and Sexuality, 1(17), 188-204. https://doi.org/10.1080/13691058.2018. 1459846.

Katzuny, K., \& Green, R.-J. (2013). The Katzuny and Green Same-Sex Marriage Scale. San Francisco, CA: Alliant University.

Kuznetsova, A., Brockhoff, P. B., \& Christensen, R. H. B. (2017). lmerTest package: Tests in linear mixed effects models. Journal of Statistical Software, 82(13), 1-26.

Merino, S. M. (2013). Contact with gays and lesbians and same-sex marriage support: The moderating role of social context. Social Science Research, 42(4), 1156-1166. https://doi.org/10.1016/j. ssresearch.2013.02.00.

Pacilli, M. G., Taurino, A., Jost, J. T., \& van der Toorn, J. (2011). System justification, right-wing conservatism, and internalized homophobia: Gay and lesbian attitudes toward same-sex parenting in Italy. Sex Roles, 65(7-8), 580-595. https://doi.org/10.1007/s11199-0119969-5.

Pereira, A., Monteiro, M. B., \& Camino, L. (2009). Social norms and prejudice against homosexuals. Spanish Journal of Psychology, 12(2), 576-584.

Pettigrew, T. F., \& Tropp, L. R. (2008). How does intergroup contact reduce prejudice? Meta-analytic tests of three mediators. European Journal of Social Psychology, 38(6), 922-934. https://doi.org/10. 1002/ejsp.504.

Prendergast, S., \& MacPhee, D. (2018). Family resilience amid stigma and discrimination: A conceptual model for families headed by same-sex parents. Family Relations, 67(1), 26-40. https://doi.org/ 10.1111/fare. 12296

Rosseel, Y. (2012). Lavaan: An R package for structural equation modeling and more. Version 0.5-12 (BETA). Journal of Statistical Software, 48(2), 1-36.

Rubio, B., Vecho, O., Gross, M., Van Rijn-Van Gelderen, L., Bos, H., Ellis-Davies, K., ... \& Lamb, M. E. (2017). Transition to parenthood and quality of parenting among gay, lesbian and heterosexual couples who conceived through assisted reproduction. Journal of Family Studies, 1-19.

Salvati, M., Ioverno, S., Giacomantonio, M., \& Baiocco, R. (2016). Attitudes toward gay men in an Italian sample: Masculinity and sexual orientation make a difference. Sexuality Research and Social Policy, 13(2), 109-118. https://doi.org/10.1007/s13178016-0218-0.

Sherif, M., \& Sherif, C. W. (1964). Reference groups. New York, NY: Harper \& Row.

Sherkat, D. E., De Vries, K. M., \& Creek, S. (2010). Race, religion, and opposition to same-sex marriage. Social Science Quarterly, 91(1), 80-98. https://doi.org/10.1111/j.1540-6237.2010.00682.x.

Simon, B., \& Klandermans, B. (2001). Politicized collective identity: A social psychological analysis. American Psychologist, 56(4), 319331.

Tanaka, J. S. (1993). Multifaceted conceptions of fit in structural equation models. In K. A. Bollen \& J. S. Long (Eds.), Testing structural equation models (pp. 10-39). Newbury Park, CA: Sage.

Vecho, O., Gross, M., Gratton, E., D’Amore, S., \& Green, R.-J. (2018). Attitudes toward same-sex marriage and parenting, ideologies and social contacts: The mediation role of sexual prejudice moderated by gender. Sexuality Research and Social Policy, 16(1), 44-57. https:// doi.org/10.1007/s13178-018-0331-3.

Vezzali, L., Capozza, D., Mari, S., \& Hichy, Z. (2007). Contact models and intergroup relations in an Italian area bordering on Austria. 
Testing, Psychometrics, Methodology in Applied Psychology, 14, 115.

Vezzali, L., \& Stathi, S. (Eds.). (2017). Intergroup contact theory. London, England: Routledge.

Vezzali, L., Turner, R., Capozza, D., \& Trifiletti, E. (2018). Does intergroup contact predict personality? A longitudinal study on the bidirectional relationship between intergroup contact and personality traits. European Journal of Social Psychology, 48, 159-173 Electronic ISSN: 1099-0992.

Webb, S., \& Chonody, J. (2014). Heterosexual attitudes toward same-sex marriage: The influence of attitudes toward same-sex parenting. Journal of GLBT Family Studies, 10(4), 404-421. https://doi.org/ 10.1080/1550428X.2013.832644.

Webb, S., Chonody, J., \& Kavanagh, P. (2017). Attitudes toward samesex parenting: An effect of gender. Journal of Homosexuality, 64(11), 1583-1595. https://doi.org/10.1080/00918369.2016. 1247540.
Whitehead, A. (2018). Homosexuality, religion, and the family: The effects of religion on Americans' appraisals of the parenting abilities of same-sex couples. Journal of Homosexuality, 65(1), 42-65. https://doi.org/10.1080/00918369.2017.1310550.

Whitley, B. E. (2009). Religiosity and attitudes toward lesbians and gay men: A meta-analysis. The International Journal for the Psychology of Religion, 19(1), 21-38. https://doi.org/10.1080/ 10508610802471104.

Wills, G., \& Crawford, R. (2000). Attitudes toward homosexuality in Shreveport-Bossier City, Louisiana. Journal of Homosexuality, 38(3), 97-116. https://doi.org/10.1300/J082v38n03_06.

Publisher's Note Springer Nature remains neutral with regard to jurisdictional claims in published maps and institutional affiliations. 